P1.52 RESISTANCE, CLONALITY AND CLINICAL ASPECTS ASSOCIATED WITH STAPHYLOCOCCUS AUREUS SAMPLES ISOLATED FROM COLONISATION SITES OF PAEDIATRIC AND ADOLESCENT PATIENTS INFECTED BY HUMAN IMMUNODEFICIENCY VIRUS

${ }^{1}$ Souza Gf, ${ }^{2} \mathrm{D}$ Ferreira, ${ }^{3} \mathrm{~F}$ Cavalcante, ${ }^{4} \mathrm{G}$ Souza, ${ }^{5} \mathrm{C}$ Hofer, ${ }^{1} \mathrm{~K}$ Santos. ${ }^{1} / \mathrm{ns}$ tituto De Microbiologia Paulo De Goes $€$ - UFRJ, Rio De Janeiro - RJ, Brazil; ${ }^{2}$ Prof. de Microbiologia, Unesa e Uva $€-R J$, Rio De Janeiro - Rj, Brazil; ${ }^{3}$ Prof. Adjunta de Microbiologia - Polo Macaé $€$ - UFRJ, Macaé - RJ, Brazil; ${ }^{4}$ Enfermeiro - UNIABEU, Belford Roxo - RJ, Brazil; ${ }^{5}$ Departamento de Medicina Preventiva, UFRJ, Rio De Janeiro - RJ, Brazil

10.1136/sextrans-2017-053264.157

Introduction HIV- infected patients are in a high-risk group to develop Staphylococcus aureus infections. From $6 \%$ to $20 \%$ of these individuals have presented colonisation by methicillin resistant isolates (methicillin resistant S. aureus "MRSA"). In addition, $S$. aureus isolates may carry genes encoding the Panton Valentine leukocidin (PVL), responsible for lysing leukocytes. This study aimed to detect and characterise $S$. aureus isolates from nares, oropharynx and saliva from paediatric and adolescent patients infected with HIV enrolled in a public paediatric outpatient clinic, between 2014 and 2015 .

Methods The $S$. aureus identification was conducted after cultivation of specimens on mannitol salt agar, using conventional tests. The determination of antimicrobial susceptibility was performed by disk diffusion test, while the minimum inhibitory concentration (MIC) by the E-test was evaluated for mupirocin. PCR was used to detect the PVL genes and to determine the SCCmec types, while the PFGE technique was used for analysis of clonality.

Results Among 100 patients included in the study, 68 (68\%) presented S. aureus colonisation, and $15(22 \%)$ of them were colonised by MRSA isolates. Colonisation by MRSA isolates was detected in the nares $(17,6 \%)$, saliva $(10,2 \%)$ and oropharynx $(8,8 \%)$ of the patients. Among the 107 s. aureus isolates, the highest percentage of resistance was $26.1 \%$ for erythromycin, followed by cefoxitin (23.4\%), gentamicin (4.7\%) and teicoplanin (3.7\%). All MRSA isolates carried the SCCmec IV and the PVL genes were found in 26 isolates of $20(29.4 \%)$ patients. The isolates were included in 11 genotype profiles.

Conclusion The study confirms the high frequency of colonisation by $S$. aureus in paediatric and adolescent patients with HIV. Besides the high rate of colonisation in nostril over than a third of the patients presented colonisation in the oropharynx and/or saliva, important aspects to be considered in the control and prevention of infections caused by $S$. aureus isolates in HIV-positive individuals.

\section{P1.53 MICRORNA EXPRESSION INDUCED BY VAGINAL MICROBIOTA CONTROLS CELL PROLIFERATION}

${ }^{1}$ Steven Smith, ${ }^{2}$ Mike Humphrys, ${ }^{2}$ Pawel Gajer, ${ }^{2}$ Shaikh lqbal, 'Vonetta Edwards, ${ }^{1}$ Jacques Ravel. ${ }^{1}$ University of Maryland, Baltimore, USA; ${ }^{2}$ Institute For Genome Sciences, Baltimore, USA

\subsection{6/sextrans-2017-053264.158}

Introduction Bacterial Vaginosis (BV) is a condition of the human vagina characterised in part by a paucity of Lactobacillus spp. and the presence of a wide array of strict and facultative anaerobes such as Gardnerella vaginalis and Atopobium vaginae. $\mathrm{BV}$ is associated with the acquisition of sexually transmitted infections such as HIV and Chlamydia trachomatis. Host microRNAs (miRNAs) are an uncharacterized factor that may control host cellular responses to Lactobacillus and BVassociated bacterial communities. Understanding the molecular mechanisms that drive or are induced by $\mathrm{BV}$-associated vaginal microbiota may help identify targets and develop strategies to restore a healthy vaginal state, which would concurrently reduce the risk of STI acquisition. We hypothesised that specific miRNAs are associated with Lactobacillus-dominated and BV-associated Community State Types (CST) by affecting specific host functions.

Methods Leveraging prospectively collected daily vaginal swab samples, the types and abundance of human miRNAs were used to gain insight into host regulatory mechanisms that potentially associate with vaginal microbial community composition shifts using miRNAseq. Random Forest miRNA feature ranking was used to identify miRNAs correlated with types of vaginal microbiota. Additional in vitro cell culture experiments were performed to demonstrate the relationship between miRNA expression, vaginal bacterial culture supernatants and epithelial cell proliferation using qPCR and Western blots.

Results miRNASeq was performed on 100 samples from 16 unique subjects in 3 longitudinal microbiota profile groups. One of the most significant miRNAs associated with BV was miR-193b. In vitro, its expression correlated with decreased cell proliferation in cells exposed to Lactobacillus spp. culture media relative to $G$. vaginalis culture supernatants.

Conclusion miR-193b over expression is associated with reduced cell proliferation in non-BV samples. Control of cell proliferation could contribute to reducing the risk of STI in Lactobacillus dominated vaginal microbiota.

\section{P1.54 DEVELOPMENT AND EVALUATION OF IN- HOUSE MULTIPLEX REAL-TIME PCR FOR DETECTION OF NEISSERIA GONORRHOEAE, CHLAMYDIA TRACHOMATIS AND MYCOPLASMA GENITALIUM INFECTION IN INFERTILITY PATIENTS}

${ }^{1}$ Sunil Sethi, ${ }^{1}$ Amit Roy, ${ }^{1}$ Rakesh Yadev, ${ }^{1}$ Rajneesh Dadwal, ${ }^{2}$ Anuradha Chakraborti ${ }^{3}$ Lakhbir Dhaliwal. 'Department of Medical Microbiology, Pgimer, Chandigarh, India; ${ }^{2}$ Department of Experimental Medicine and Biotechnology, Pgimer, Chandigarh, India; ${ }^{3}$ Department of Obstetrics and Gynaecology, Pgimer, Chandigarh, India

\subsection{6/sextrans-2017-053264.159}

Introduction: C.trachomatis, N.gonorrhoeae and M.genitalium are important cause of infertility but detection is usually by PCR which has to be performed indvidually for each pathogen.The aim of this study was to develop In house multiplex Real time PCR assay for simultaneous detection of all these pathogens in single run and will also help in detecting co infecion if present thus saving cost and time in cases of infertility.

Methods The Taqman probe based multiplex qPCR for detection of C.trachomatis, N.gonorrhoeae and M.genitalium was developed using different primers and probes. Analytical sensitivity of multiplex qPCR was determined using pGEMT Easy vector cloned with target genes. The detection limit for each organism was determined using 10 fold dilutions of targets. The multiplex qPCR was evaluated in 248 clinical samples i.e 98 infertile (endometrial biopsy and endocervical swabs) and 150 healthy controls (endocervical swabs). The sensitivity, specificity, positive and negative predictive value (PPV and NPV) of multiplex qPCR was calculated. 
Results The sensitivity, specificity, PPV and NPV of new multiplex qPCR was $98.80 \%, 100 \%, 100 \%$ and $99.69 \%$ respectively compared to uniplex qPCR. The discordant result of multiplex qPCR was detected in 1 sample. Developed multiplex qPCR showed 100\% sensitivity, specificity, PPV and NPV for C.trachomatis and N.gonorrhoeae respectively. The sensitivity, specificity, PPV and NPV for M.genitalium were $97.78 \%$, $100 \%, 100 \%$ and $99.72 \%$ respectively. No cross-reactions were detected between target organisms or with related species.

Conclusions Multiplex In house qPCR in this study has shown high sensitivity and specificity for detection of C.trachomatis, N.gonorrhoeae and M.genitalium in infertility patients which facilitate the opportunity to be used as a rapid diagnostic tool and for initiation of early treatment in resource poor settings where syndromic approach is being followed. This assay needs to be performed on the larger sample size and using different specimens prior to large-scale screening.

\section{P1.55 DETECTION OF GENITAL MYCOPLASMAS IN WOMEN VISITING THE INFERTILITY CLINIC OF AN ACADEMIC HOSPITAL, PRETORIA, SOUTH AFRICA}

${ }^{1}$ Thabang C Duba, ${ }^{2}$ Remco Ph Peters, ${ }^{3}$ Marthie M Ehlers, ${ }^{1}$ Noëlle Pruis, ${ }^{1}$ Sweetness M Majola, ${ }^{3}$ Marleen Kock. 'University of Pretoria, Pretoria, South African Republic; ${ }^{2}$ University of Pretoria/Anova Health Institute, Pretoria, South African Republic; ${ }^{3}$ University of Pretora/National Health Laboratory Service, Pretoria, South African Republic

\subsection{6/sextrans-2017-053264.160}

Introduction Sexually transmitted infections (STIs) continue to be a significant public health problem with a high burden in women of reproductive age. Rates of Chlamydia trachomatis, Neisseria gonorrhoeae and Trichomonas vaginalis are frequently tested for and rates of infection are generally high in African settings, but the prevalence of other genital STIs is largely unknown. The aim of this study was to determine the prevalence of genital mycoplasmas (Mycoplasma genitalium, $M$. hominis, Ureaplasma parvum and U. urealyticum) in women visiting the infertility clinic of a tertiary academic hospital in South Africa.

Methods In this pilot evaluation self-collected vaginal swabs were obtained from 51 women visiting the infertility clinic. The genomic DNA was extracted from the swabs using the ZR Fungal/Bacterial DNA Miniprep (Thermo Scientific, USA) and analysed using the Anyplex II STI-7 (Seegene, Korea) real-time PCR assay for the simultaneous detection and identification of seven STIs including the four mycoplasma species.

Results The real-time PCR assay detected the following genital mycoplasmas and co-infections in the 51 women: U. parvum [55\% (28/51)], M. hominis [20\% (10/51)] and U. urealyticum [16\% (8/51)]; none of the specimens tested positive for $M$. genitalium. Among the nine patients where mixed infections were observed, M. hominis and Ureaplasma spp. were frequently detected together $[67 \%$ (6/9)]. In addition to the mycoplasmas, one woman tested positive for C. trachomatis; $N$. gonorrhoeae and T. vaginalis were not detected.

Conclusion This pilot study demonstrated an unexpectedly high rate of genital mycoplasma infections among women visiting an infertility clinic. The burden of genital mycoplasma infection is largely unknown and warrants further investigation, in particular with regards to the prevalence and clinical significance in different population groups.

Support: Anyplex II STI-7 kits provided by Seegene, Korea

\section{P1.56} PREDICTORS OF CARDIOVASCULAR RISK AND
ATHEROGENIC INDICES AMONG ADULT HIV
SEROPOSITIVE PATIENTS ON HIGHLY ACTIVE
ANTIRETROVIRALS IN WESTERN NIGERIA: A CASE-
CONTROL STUDY

Usman Saheed Opeyemi, Agboola Ganiyu. Department of Clinical Laboratory Services, Equitable Health Access Initiative, Lagos, Nigeria

\subsection{6/sextrans-2017-053264.161}

Introduction Cardiovascular risk factors place HIV-infected patients at increased risk for cardiovascular diseases (CVDs) due to complex interactions between traditional CVD risk factors, antiretroviral therapy (ART) and HIV infection itself. The report of the 2012 National Reproductive Health Survey Plus indicated that the prevalence of HIV/AIDS in Nigeria is about $3.4 \%$ while Ondo State has a prevalence of $4.3 \%$. This study was therefore designed to evaluate the CD4+ T-cell count, atherogenic indices and risk score of adult HIV seropositives on Highly Active Antiretroviral Therapy (HAART), those not yet started on HAART and HIV seronegative control subjects. Hypothesis tested was the effect of the various drugs on the indices determining the risk level.

Methods Serum levels of CD4+ cell count of adult HIV seropositive subjects on HAART, HAART naïve subjects and seronegative controls were determined using flow cytometry while their atherogenic indices and Framingham risk score were determined from enzymatic spectrophotometrically determined lipids and lipoproteins. Ethical approval was obtained from the Ondo State Ministry of Health Research Ethics Committee, Akure, Nigeria. All data were expressed as Mean \pm Standard Deviation and analysed with Analysis of Variance (ANOVA) while multiple comparisons were done using Post Hoc Bonferonni test.

Results The average duration (in months) of the use of HAART in the group 1 subjects is $25.63 \pm 19.99$ while the average duration (in months) of cotrimoxazole use for subjects in group 2 is 7.10 \pm 4.89 . There was a significant mean increased weight in the control subjects as compared with that of the other two groups. The mean serum cardiac risk ratio (CRR), atherogenic index of plasma (AIP), atherogenic coefficient (AC) and Framingham Risk Score (FRS) were significantly increased in the HAART group as compared with those of the two other groups.

Conclusion HIV appears to have negatively altered the exogenous and endogenous synthesis and metabolism of lipids and lipoproteins in the liver, with ultimate effect on the atherogenic indices and risk score. This is worsened by antiretroviral therapy as the increased levels of these indices were mainly seen in the HAART group, constituting a major risk for cardiovascular diseases in these patients, thus increasing mortality rate.

\section{P1.57 HOW TO FACILITATE AND IMPROVE SCREENING OF SEXUALLY-TRANSMITTED INFECTIONS IN WOMEN POPULATION}

Vittorio Sambri, Giorgio Dirani, Patrizia Farabegoli. Unit of Microbiology, The Great Romagna Hub Laboratory, Cesena - Italy

\subsection{6/sextrans-2017-053264.162}

Introduction Sexually Transmitted Infections (STIs) are increasing worldwide. Innovative approaches are required to eliminate barriers to STIs testing such as home-based self-sampling for patients that are difficult to reach or for whose that refuse 